\title{
CELL-LIKE MAPPINGS BETWEEN CS SETS
}

\author{
JAMES P. HENDERSON
}

\begin{abstract}
A CS set is a certain type of stratified metric space. The structure of a CS set which is a generalized $n$-manifold is determined. A class of cell-like mappings between CS sets is defined, and it is shown that such a cell-like mapping with manifold image $M^{n}, n \neq 4$, is approximable by homeomorphisms.
\end{abstract}

Introduction. Edwards' approximation theorem [4] for CE mappings from an $n$-manifold $M, n \geqslant 5$, onto a finite-dimensional ANR has provided an elegant, yet simple, technique for identifying those mappings which can be approximated by homeomorphisms. In attempting to extend and exploit this result, it seems natural to consider situations which lead one to reduce given problems to ones involving $\mathrm{CE}$ mappings from manifolds onto finite-dimensional ANR's. In the cases to be considered here, we will be working with finite-dimensional metric spaces which are stratified into topological manifolds. The main thrust will be to define a certain class of cell-like mappings between such stratified spaces and to consider the situation in which at least one of the domain and range is a manifold.

Related results were first obtained by Handel [5] and then, closer to the spirit of the present problem, the author [7-10]. In the previous results, each point preimage was required to be individually shrinkable, a condition not required here.

1. Preliminaries. A (TOP) stratified space $X$ consists of a topological space $X$ and a filtration of $X$ into closed subsets $X^{(k)}$ of $X$ such that $\varnothing=X^{(-1)} \subseteq X^{(0)} \subseteq X^{(1)} \subseteq$ $\cdots \subseteq X$ and the components of $X[k]=X^{(k)}-X^{(k-1)}$ are open subsets of $X[k]$ (and are topological $k$-manifolds without boundary). The set $X[k]$ is the $k$-stratum and $X^{(k)}$ is the $k$-skeleton.

A $C S$ set $X$ is a TOP stratified space $X$ such that for each integer $m$ and $x \in X[m]$, there is a compact stratified space $L$ and an open embedding $h$ : $\mathbf{R}^{m} \times c L \rightarrow X$ taking $0 \times c$ to $x$ such that

$$
h\left(\mathbf{R}^{m} \times c L\right) \cap X[k]=h\left(\mathbf{R}^{m} \times(c L)^{(k-m)}\right)=h\left(\mathbf{R}^{m} \times c\left(L^{(k-m-1)}\right)\right) .
$$

Here $c L$ is the open cone on $L$ stratified by $c=c L[0]$ and $(c L)^{n}=c\left(L^{(n-1)}\right)$. We assume all spaces are finite dimensional, so there is an integer $n$ such that $X=X^{(n)}$.

There may be several CS structures on a given topological space $X$. However, one may obtain a minimal CS stratification on a CS set as follows. Let $I(x, X)$ be the intrinsic dimension of $x$ in $X$, defined by $I(x, X)=\max \{n \in \mathbf{Z} \mid$ there is a compact

Received by the editors February 23, 1983. Presented to the Society, November 13, 1982. 1980 Mathematics Subject Classification. Primary 57N80, 57N15; Secondary 57P99, 54E20. Key words and phrases. CS set, cell-like, generalized manifold.

c1984 American Mathematical Society $0002-9939 / 84 \$ 1.00+\$ .25$ per page 
space $L$ and an open embedding $h: \mathbf{R}^{n} \times c L \rightarrow X$ taking $0 \times c$ to $\left.x\right\}$. If $X^{(n)}=\{x \in$ $X \mid I(x, X) \leqslant n\}$ and $X[n]=X^{(n)}-X^{(n-1)}$, then according to Handel [6], $\left\{X^{(k)}\right\}$ does provide a CS structure for a CS set $X$, and if $\left\{S^{(k)}\right\}$ is any other CS structure in $X, X^{(n)} \subseteq S^{(n)}$. Thus any CS structure mentioned will be assumed to be the minimal one.

2. Mappings between CS sets. The problem to be considered is that of determining conditions under which proper, cell-like (CE) mappings between CS sets may be approximated by homeomorphisms. We first consider some restrictions on the point preimages of such a CE mapping. The following definition is taken from [7] and applied to CS sets.

Definition. A compact subset $C$ of a CS set $X$ is cellular in $X$ if there is a pseudoisotopy $h_{t}: X \rightarrow X$ such that $C$ is the only nondegenerate point preimage of $h_{1}$.

The nature of cellular subsets of CS sets is described by the following theorem. The proof is identical to that of Theorem 2.2 of [7] and is omitted.

THEOREM 1. Let $C$ be a compact subset of the CS set $X$. Then the following are equivalent:

(1) $C$ is a cellular subset of $X$.

(2) $\pi: X \rightarrow X / C$ is approximable by homeomorphisms.

(3) $X=\cap_{i=1}^{\infty} N_{i}$ where $N_{i}$ is a neighborhood of $C$ homeomorphic to $\mathbf{R}^{k} \times c L$ for some compact stratified space $L$.

It is not our intention to discuss CE mappings between CS sets having cellular point preimages, for the results on cellular mappings between polyhedra in [7-10] may be generalized to apply to CS sets with the aid of Theorem 2 below. However, at this stage we note that if $f: X \rightarrow Y$ is a CE mapping between CS sets which is approximable by homeomorphisms, it follows from part (3) of the above theorem that each point preimage would be a cellular subset of $X$. Our restriction on point preimages will then consist of some property of cellular sets, with the chosen property not strong enough to guarantee cellularity. Note that if $C$ is a cellular subset of a CS set $X$, then for each neighborhood $U$ of $C$, there is a neighborhood $V$ of $C$ in $U$ and a stratum respecting contraction $h_{t}: V \rightarrow U$. By stratum respecting we mean that $I\left(h_{t}(x), X\right) \geqslant I\left(h_{s}(x), X\right)$ for $t \leqslant s$. Thus there is a "nice" contraction of $C$ in each of its neighborhoods. This is the property we wish to discuss.

Definition. A compact subset of $C$ of a CS set $X$ is a rooted cell-like set if for each neighborhood $U$ of $C$ in $X$, there is a neighborhood $V$ of $C$ in $X$ and a stratum respecting contraction of $V$ in $U$.

It should be pointed out that if $X$ is a manifold, there is no difference between cell-like and rooted cell-like subsets. A nontrivial example of a rooted cell-like subset of $\mathbf{R}_{+}^{3}$, the upper half-space in $\mathbf{R}^{3}$, may be obtained from Bing's [1] decomposition of $\mathbf{R}_{+}^{3}$ used to show that the identity sewing of two Alexander crumpled cubes yields $S^{3}$. One need only run an arc through the Cantor set where the nondegeneracy set of the decomposition intersects the $\mathbf{R}^{2}$ boundary of $\mathbf{R}_{+}^{3}$ and take the union of that arc in $\mathbf{R}^{2}$ and the nondegeneracy set of the decomposition. 
It also should be pointed out that rooted cell-like sets were the natural candidates to which a cellularity criterion was applied in [7], a criterion which could also be extended to CS sets.

We now define the class of mappings with which we will deal.

Definition. A proper mapping $f: X \rightarrow Y$ between CS sets is a rooted cell-like ( $R C E$ ) mapping if for each $y \in Y, f^{-1}(y)$ is a nonempty rooted cell-like subset of $X$.

3. Generalized manifolds. We shall mainly be concerned here with RCE mappings between CS sets, at least one of which is a topological manifold. Since RCE mappings are fine homotopy equivalences, if $f: X \rightarrow Y$ is a RCE mapping with either $X$ or $Y$ a generalized $n$-manifold, both $X$ and $Y$ must be generalized $n$-manifolds. Thus we wish to investigate the structure of CS sets which are generalized $n$-manifolds. We first need a lemma concerning the product of certain generalized manifolds with the real line $\mathbf{R}^{\prime}$.

LEMMA 1. Let $A$ be a closed subset of a generalized n-manifold $X, n \geqslant 4$, with $\operatorname{dim} A \leqslant n-2$. If $(X-A) \times \mathbf{R}^{1}$ is an $(n+1)$-manifold, then $X \times \mathbf{R}^{\mathbf{1}}$ is also an $(n+1)$-manifold.

Proof. Let $f: M \rightarrow X$ be a resolution of $X$ [11]. Then if $G$ is the decomposition of $M$ whose nondegeneracy set consists of $\left\{f^{-1}(x) \mid x \in A\right\}$, it follows that $(M / G) \times$ $\mathbf{R}^{1} \cong M \times \mathbf{R}^{1}$ since $\operatorname{dim} A \leqslant n-2$ [3]. Thus $f \times$ id: $M \times \mathbf{R} \rightarrow X \times \mathbf{R}$ can be approximated by a cell-like mapping $g: M \times \mathbf{R} \rightarrow X \times \mathbf{R}$ which is 1-1 over $A$. Since $A$ is closed and $(X-A) \times \mathbf{R}^{1}$ is an $(n+1)$-manifold, one can then approximate $g \mid g^{-1}((X-A) \times \mathbf{R})$ by a homeomorphism $\tilde{g}$ which may be extended to $g^{-1}((X-A) \times \mathbf{R})$ by $g$.

The following characterization of $\mathrm{CS}$ sets which are generalized $n$-manifolds is very similar to Cannon's result for polyhedra [2].

THEOREM 2. Let $X$ be a CS set which is a generalized n-manifold. Then $X=X[n] \cup$ $X[0]$. If $n \leqslant 3, X[0]=\varnothing$ and for $n \geqslant 5$, each $x \in X[0]$ has a neighborhood homeomorphic to the open cone over a non-simply-connected homology $(n-1)$-sphere.

Proof. Since for each $x \in X[k], x$ has an open neighborhood homeomorphic to $\mathbf{R}^{k} \times c L$, it suffices to classify the possible structures of these defining neighborhoods. $\mathbf{R}^{k} \times c L$ will also be a generalized $n$-manifold, as will $\left(\mathbf{R}^{k} \times c L\right)-\left(\mathbf{R}^{k} \times c\right)$ $\cong \mathbf{R}^{k+1} \times L$. Therefore $c L$ is a generalized $(n-k)$-manifold and $L$ is a generalized $(n-k-1)$-manifold with the global homology of an $(n-k-1)$-sphere. Thus if $\operatorname{dim} c L \leqslant 3, \operatorname{dim} L \leqslant 2$, and $L$ is a topological $(n-k-1)$-sphere, $c L$ is an $(n-k)$ manifold, and $x \in X[n]$.

We now consider the case where $\operatorname{dim} c L \geqslant 4$. If $k \geqslant 2$, then $\operatorname{dim} \mathbf{R}^{k} \times c L \geqslant 6$ and $\mathbf{R}^{k} \times c L$ is a generalized $n$-manifold satisfying the disjoint disks property [3] and hence is an $n$-manifold [4].

For $k=1$ we note that, as above, $\left(\mathbf{R}^{1} \times c L\right)-\left(\mathbf{R}^{1} \times c\right)$ is homeomorphic to $\mathbf{R}^{2} \times L$ and hence is an $n$-manifold since $\operatorname{dim} \mathbf{R}^{2} \times L \geqslant 5$. Thus we can now apply Lemma 1 with $X=c L$ and $A=c$ to conclude that $\mathbf{R}^{1} \times c L$ is an $n$-manifold. Thus it follows that $X[k]=\varnothing$ for $0<k<n$. 
For the remaining case we have $k=0$. Here $x \in X[0]$ has a neighborhood homeomorphic to $c L$. Again, $L$ has the global homology of an $(n-1)$-sphere, but $L$ need not be an $(n-1)$-manifold. Note that since $c L-c$ corresponds to an open subset of $X-X[0]=X[n], c L-c \cong \mathbf{R}^{1} \times L$ is an $n$-manifold, and $L$ is a generalized $(n-1)$-manifold. Thus for $n \geqslant 5$, there is an $n$-manifold $M$ and a $C E$ mapping $f: M \rightarrow L$ [11]. Now $f \times$ id: $M \times \mathbf{R} \rightarrow L \times \mathbf{R}$ can be approximated arbitrarily closely by homeomorphisms since both $M \times \mathbf{R}$ and $L \times \mathbf{R}$ are $n$-manifolds [4]. Thus one can then extend a homeomorphism $h$ : $c M-c \rightarrow c L-c$ to a homeomorphism $\tilde{h}: c M \rightarrow c L$, and $M$ is the desired homology $(n-1)$-sphere.

The next two corollaries follow easily.

COROllary 1. If $X$ is a CS set which is a generalized n-manifold, then $X \times \mathbf{R}$ is an n-manifold.

COROLlaRY 2. If $X$ and $Y$ are $C S$ sets with either $X$ or $Y$ a generalized n-manifold and $f: X \rightarrow Y$ a $C E$ mapping, then $f \times \mathrm{id}: X \times \mathbf{R} \rightarrow Y \times \mathbf{R}$ is approximable by homeomorphisms.

4. RCE mappings and manifold. Examples have long been known of RCE mappings with manifold domain which cannot be approximated by homeomorphism. One can produce such an example by collapsing to a point a contractible submanifold $M^{n}$ of $S^{n}, n \geqslant 5$, where the boundary of $M^{n}$ is a non-simply-connected homology $(n-1)$-sphere. It follows from Theorem 2 that such isolated singularities are the only possibilities since the image of a manifold under an RCE mapping is a CS set which is a generalized $n$-manifold having only discrete singularities in its 0 -stratum.

Although one can create new singularities with an RCE mapping, the following result states that singularities in a CS set which is a generalized $n$-manifold cannot be eliminated by RCE mappings.

THEOREM 3. Let $f: X \rightarrow M$ be an RCE mapping with $M$ an $n$-manifold, $n \neq 4$. Then $f$ can be approximated by homeomorphisms.

Proof. Since $X$ must be an $n$-manifold for $n \leqslant 3$, we need only consider the case $n \geqslant 5$. Since $X=X[n] \cup X[0]$, the proof will be completed by showing that $X[0]=$ $\varnothing$. In any case, $X[0]$ is a discrete subset of $X$ and we may assume $f$ is $1-1$ over $M-f(X[0])$.

Let $x \in X[0]$. Then $x$ has a neighborhood homeomorphic to $c H$, where $H$ is a homology $(n-1)$-sphere. Given a neighborhood $U$ of $f^{-1}(f(x))$, there is a neighborhood $V$ of $f^{-1}(f(x))$ in $U$ missing $X[0]-x$ and a stratum respecting contraction $h_{t}$ : $V \rightarrow U$. Choose a tame $n$-cell $D$ in $f(V)$ containing $f(x)$ in its interior. Then $f^{-1}(\partial D)$ is a bicollared $(n-1)$-sphere $\Sigma$ in $X[n] \cap V$. Using the cone neighborhood $c H$ and the special contraction $h_{t}$, identify an $n$-manifold $W$ in $X[n] \cap Y$ with two boundary components $M_{1}$ and $M_{2}$ such that $M_{1}=\Sigma, M_{2}=H$, and $W$ strong deformation retracts onto $M_{2}$. Let $r: M_{1} \rightarrow M_{2}$ be the final stage of the strong deformation retraction restricted to $M_{1}$. Then $r: M_{1} \rightarrow M_{2}$ is a degree-1 mapping. If $\tilde{H}$ is the universal cover of $M_{2}$, the mapping $r$ lifts to $\tilde{r}: M_{1} \rightarrow \tilde{H}$. However, if $\pi_{1}\left(M_{2}\right)=\pi_{1}(H)$ 
is finite, then $\operatorname{deg} p=k$ for some integer $k$, where $p: \tilde{H} \rightarrow M_{2}$ is the covering map. But since $1=\operatorname{deg} r=(\operatorname{deg} \tilde{r})(\operatorname{deg} p), \operatorname{deg} p$ can be no integer other than one. Thus $\pi_{1}(H)$ must be infinite. However, this implies $H_{n-1}(\tilde{H})=0$ and $\operatorname{deg} r=0$. Thus a contradiction is reached in either case and the assumption that $x \in X[0]$ is incorrect.

\section{REFERENCES}

1. R. H. Bing, A homeomorphism between the 3-sphere and the sum of two solid horned spheres, Ann. of Math. (2) 56 (1952), 354-362.

2. J. W. Cannon, The recognition problem: what is a topological manifold, Bull. Amer. Math. Soc. 84 (1978), 832-866.

3. R. J. Daverman, Detecting the disjoint disks property, Pacific J. Math. 93 (1981), 277-298.

4. R. D. Edwards, Approximating certain cell-like maps by homeomorphisms (manuscript).

5. M. Handel, Approximating stratum preserving $C E$ maps between $C S$ sets by stratum preserving homeomorphisms, Geometric Topology (L. C. Glaser and T. B. Rushing, eds.), Lecture Notes in Math., Vol. 438, Springer-Verlag, New York, 1975, pp. 245-250.

6. A resolution of stratification conjectures concerning CS sets, Topology 17 (1978), 167-175.

7. J. P. Henderson, Cellularity in polyhedra, Topology Appl. 12 (1981), 267-282.

8. _ A cellular map between nonhomeomorphic polyhedra whose nondegeneracy set is a null sequence, Proc. Amer. Math. Soc. 85 (1982), 447-450.

9. Cellular maps between polyhedra, Trans. Amer. Math. Soc. 272 (1982), 525-537.

10. __ Approximating cellular maps between low dimensional polyhedra, Pacific J. Math. 101 (1982), $321-331$.

11. F. Quinn, Resolutions of homology manifolds, and the topological characterization of manifolds, preprint.

Department of Mathematics, Texas A \& M University, College Station, Texas 77843 